\title{
A New Structural Model for Collagen
}

\author{
Kenji Okuyama, ${ }^{*}$ Motowo Takayanagi, ${ }^{*}$ Tamaichi Ashida, ${ }^{* *}$ \\ and Masao KAKUDo*** \\ *Department of Applied Chemistry, Kyushu University, Fukuoka 812, Japan. \\ **Department of Applied Chemistry, Nagoya University, Nagoya 464, Japan. \\ ***Institute for Protein Research, Osaka University, Osaka 565, Japan.
}

(Received February 10, 1977)

KEY WORDS Collagen / Molecular Structure / X-Ray Analysis /
(Pro-Pro-Gly) $)_{10} /$

During the last half century, following the pioneer work of Herzog and Jancke, ${ }^{1}$ the structure of collagen has been investigated by using the X-ray diffraction method, and several structural models have been proposed. The general features of the wide angle X-ray diffraction patterns of native collagen reported by many researchers were as follows: ${ }^{2}$

(1) a strong meridional arc at $2.86 \AA$,

(2) layer lines at 9.5 and $4.0 \AA$, each with a pair of off meridional spots,

(3) a diffuse cloud of density on the equator around $4.5 \AA$,

(4) clouds of density above and below this equatorial group,

(5) a very dense moisture-sensitive equatorial reflection at about $11 \AA$.

Two possible ways have been found to assign the layer line indices to all the above reflections except (4), whose diffraction was too vague and broad to be assigned an index. As shown in Table I, one way was to take $20 \AA$ as the pseudo-period along the fiber axis and another way was to take $28.6 \AA$ as the pseudo-period. The term "pseudo-period" is used here, since collagen is not a polymer with a simple repeating unit, in view of its amino acid sequence. In order to assign the 0th Bessel order to the meridional reflection of spacing $2.86 \AA$ and the first Bessel order to the layer lines of spacings 4.0 and $9.5 \AA$, the collagen molecule must be a $7 / 2$ helix, which has 7 scattering units and 2 turns in the pseudo-period of $20 \AA$, or a $10 / 3$ helix which has 10 scattering units and 3 turns
Table I. Two possible layer line indexings for the X-ray fiber diagram of collagen

\begin{tabular}{lcrr}
\hline \multirow{2}{*}{$\begin{array}{l}\text { Observed } \\
\text { spacings }\end{array}$} & \multicolumn{2}{c}{ Layer index } & $\begin{array}{c}\text { Bessel } \\
\text { order }\end{array}$ \\
\cline { 2 - 3 } & $c=20.0 \AA$ & $c=28.6 \AA$ & \\
\hline $2.86 \AA$ & 7 & 10 & 0 \\
4.0 & 5 & 7 & -1 \\
9.5 & 2 & 3 & 1 \\
$\infty$ & 0 & 0 & 0 \\
\hline
\end{tabular}

in the pseudo-period of $28.6 \AA$. Radial projections, or helical nets, of these two helices are shown in Figure 1. An open circle indicates a representative position in a scattering unit, for example, a position of an $\mathrm{N}$ atom of any amino acid residue. Both structures have one scattering unit per $2.86 \AA$ as we see in Figure 1; Pauling and Corey ${ }^{3}$ suggested by considering the measured and calculated density that collagen had three amino acid residues per $2.86 \AA$ along the fiber axis. Accordingly one scattering unit must contain three amino acid residues. The arrows and letters indicate possible directions of chemical bonds. Although various structures having different numbers of peptide strands can be imagined, depending on the direction of chemical bonds, we should take into account of only the structures having less than five strands because the vertical distance between the neighboring scattering units of more than four-strand structure is greater than the extended distance, about $11 \AA$, of three amino acid residues. Historically, single-strand struc- 


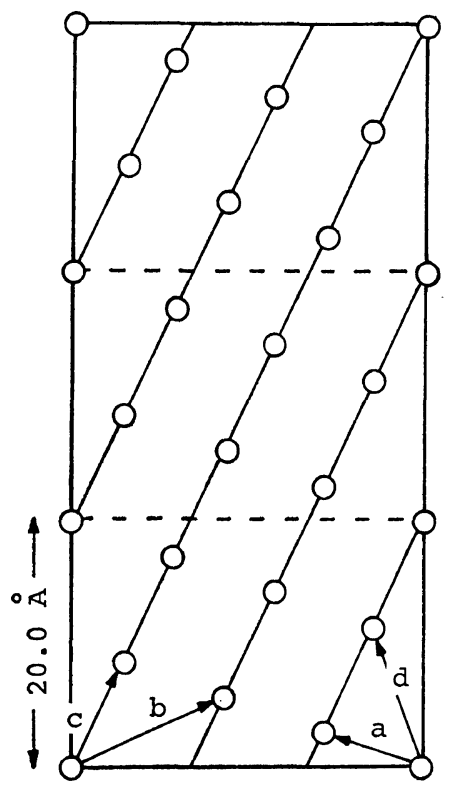

(I)

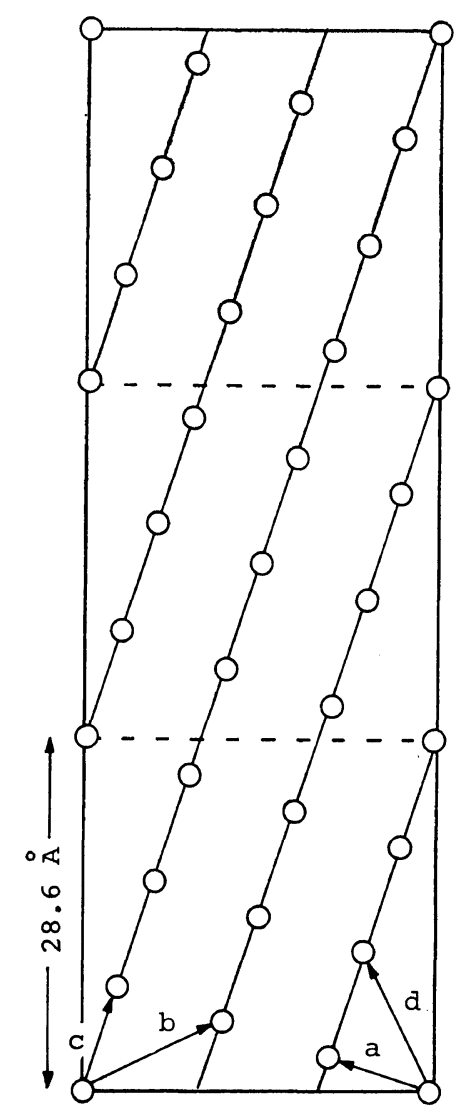

(2)

Figure 1. Two possible radial projections for collagen. The pseudo-period along the fiber axis is (1) $20.0 \AA$; (2) $28.6 \AA$.

tures of $7 / 2$ helix with pitch length $10 \AA((1)-a)$ or $10 / 3$ helix with pitch length $9.5 \AA((2)-a)$ were proposed by Cowan, North, and Randall ${ }^{4}$ and Cohen and Bear. ${ }^{5}$ Now it is obvious from several physico-chemical observations that tropocollagen contains three polypeptide chains. So only the three-strand structures ((1)-c and (2)-c) are allowed. One of the three-strand structures, in which each strand has 10 scattering units in pitch length $85.8 \AA$ (10/1 helix), is a basal structure of the currently proposed collagen model $^{6-8}$ and its modification. ${ }^{9}$ The another type of three-strand structures with three $7 / 1$ helices of pitch length $60 \AA((1)$-c) has not been proposed for any collagen model so far.

Sakakibara and his co-workers ${ }^{10}$ synthesized collagen model sequential polypeptides (Pro-Pro-
Gly $)_{n}$ ( $n=10$ or 20 ) by using a fragment condensation technique together with a Merrifield resin. The structure analysis of a single crystal of (Pro-Pro-Gly) $)_{10}$ showed that the pseudo-period along the fiber axis is $20.08 \AA$ and a "molecule" consists of three peptide strands, in which each strand has 7 scattering units (21 amino acid residues) in pitch length $60.24 \AA .{ }^{11}$ This structure corresponds to the second of the two possible three-strand structures for collagen mentioned above. Yonath and $\mathrm{Traub}^{8}$ reported that the X-ray fiber diagram of (Pro-Gly-Pro) ${ }_{n}$ was very similar to that of native collagen. On the other hand, the molecular structure of (ProGly-Pro $)_{n}$ is also expected to be very similar to that of (Pro-Pro-Gly) ${ }_{10}$, because both polypeptides have the same amino acid sequence 
except for the terminals. The precession photograph of (Pro-Hyp-Gly) 9 showed also the existence of a pseudo-period of $20 \AA .{ }^{12}$ Further, Miller and Scheraga ${ }^{13}$ calculated the conformational energy for (Pro-Pro-Gly) ${ }_{n}$ and reported that the most energetically stable conformation was close to the three-strand structure composed of $7 / 1$ helices rather than $10 / 1$ helices. In conclusion, our structural model for (Pro-Pro-Gly) 10 can be reasonably assigned as a possible model for the native collagen structure. The molecular structure of (Pro-Pro-Gly) 10 has been reported in the reference ${ }^{14}$ and the detailed structure using three-dimensional X-ray data will be published soon.

\section{REFERENCES}

1. R. O. Herzog and W. Jancke, Ber., 53, 2162 (1920).

2. R.E. Dickerson, in "The Proteins," Vol. 2, J. T. Randall, Ed., Academic Press, New York, N.Y., 1964, p 603.

3. L. Pauling and R. B. Corey, Proc. Natl. Acad. Sci. U.S., 37, 272 (1951).

4. P. M. Cowan, A. C. T. North, and J. T. Randall, in "Nature and Structure of Collagen," J.T. Randall, Ed., Academic Press, New York, N.Y., 1953, p 241.

5. C. Cohen and R. S. Bear, J. Amer. Chem. Soc., 75, 2783 (1953).

6. A. Rich and F. H.C. Crick, Nature, 176, 915 (1955).

7. G. N. Ramachandran and G. Kartha, ibid., 176, 593 (1955).

8. A. Yonath and W. Traub, J. Mol. Biol., 43, 461 (1969).

9. G. N. Ramachandran, in "Treatise on Collagen," Vol. 1, G. N. Ramachandran, Ed., Academic Press, New York, N.Y., 1967, p 103.

10. S. Sakakibara, Y. Kishida, Y. Kikuchi, R. Sakai, and K. Kakiuchi, Bull. Chem. Soc. Jpn., 41, 1273 (1968).

11. K. Okuyama, N. Tanaka, T. Ashida, M. Kakudo, S. Sakakibara, and Y. Kishida, J. Mol. Biol., 72, 571 (1972).

12. S. Bando, M. Kakudo, S. Sakakibara, and Y. Kishida, in preparation.

13. M. H. Miller and H. A. Scheraga, J. Polym. Sci., Polym. Symp., 54, 171 (1976).

14. K. Okuyama, N. Tanaka, T. Ashida, and M. Kakudo, Bull. Chem. Soc. Jpn., 49, 1805 (1976). 